\title{
Prevalence and Risk Factors for Diabetic
}

\section{Peripheral Neuropathy Among Saudi Hospitalized Diabetic Patients: A Nested Case-Control Study}

This article was published in the following Dove Press journal: International Journal of General Medicine

\author{
Fahad AS Aleidan (D) ${ }^{1,2}$ \\ Banderi A Ahmad' \\ Farah A Alotaibi iD' \\ Dalal H Aleesa (iD) \\ Nuha A Alhefdhi' \\ Motasim Badri \\ Abdel Galil Abdel Gader' \\ 'College of Medicine, King Saud Bin \\ Abdulaziz University for Health Sciences, \\ Riyadh, Saudi Arabia; ${ }^{2}$ College of \\ Medicine, King Abdulaziz Medical City, \\ Riyadh, Saudi Arabia
}

Objective: To determine the prevalence and the risk factors of diabetic peripheral neuropathy (DPN) in hospitalized adult Saudi diabetics.

Methods: This is a retrospective, nested case-control study conducted at King Abdulaziz Medical City (KAMC) in Riyadh, Saudi Arabia. All diabetic patients admitted to the hospital between the January 1, and December 31, 2018 were considered for inclusion in the study. Patients with DPN were identified and three controls per case were randomly selected from the remaining diabetic patients without peripheral neuropathy (PN).

Results: A total of 2,096 adult diabetic patients were identified during the study period. Of these, 73 patients $(3.5 \%)$ were confirmed to be suffering from DPN and 219 were included as controls. When comparing diabetic with the control cases, DPN cases were significantly older $(p=0.002)$, had a significantly higher proportion of type 2 diabetes $(p=0.023)$, chronic kidney disease $(p<0.0001)$, cerebral vascular stroke $(p=0.027)$, hypertension $(p=0.005)$, dyslipidemia $(p=0.002)$, peripheral vascular disease $(p<0.0001)$, osteoarthritis $(p=0.034)$, diabetic ketoacidosis $(p=0.003)$, foot ulcers $(p=0.006)$, gangrene $(p=0.001)$, lower limb ischemia $(p=0.001)$, increased duration with diabetic disease $(p=0.031)$, increased BMI $(p=0.003)$, higher serum creatinine $(p<0.001)$ and lower serum albumin levels $(p=0.035)$. In the multivariate logistic regression, only older age \{odds ratio (OR) 1.02, 95\% CI 1.01$1.04, p=0.031$, chronic kidney disease (OR 2.39, 95\% CI 1.23-4.64, $p=0.010$ ) and peripheral vascular disease (OR 3.14, 95\% CI 1.39-7.13, $p=0.006$ ) were independently associated with DPN.

Conclusion: This study identified several risk factors that contributed to the development of DPN in Saudis. These must be considered in strategies and campaigns aimed at risk reduction of cardiovascular and chronic diseases, and consequently progression of DPN.

Keywords: diabetes mellitus, diabetic peripheral neuropathy, risk factors
Correspondence: Fahad AS Aleidan College of Medicine, King Saud Bin Abdulaziz University for Health Sciences, Riyadh, Saudi Arabia Tel +966II80IIIII

Email faleidan@yahoo.com

\section{Introduction}

Diabetic peripheral neuropathy DPN is defined as, "the presence of symptoms and/ or signs of peripheral nerve dysfunction in patients with diabetes after the exclusion of other causes," and may be present despite a lack of symptoms. ${ }^{1}$ The diagnosis relies on clinical signs as well as quantitative electrophysiological testing: ${ }^{1}$ DPN is a common complication in patients with diabetes and its consequences can be distressing, as patients may develop neuropathic feet pain and decreased sensation that can lead to frequent falls and injuries. Patients may also develop foot ulcers. All these consequences of DPN will unavoidably end in the reduction in the quality 
of life and significant economic burden both to the patients and society. ${ }^{2-5}$ Although up to $50 \%$ of patients with DPN are asymptomatic, some patients may manifest burning feet pain, due to progressive sensory impairment, which responds poorly to analgesics. Rarely, this sensory impairment could lead to foot ulceration and eventually to lower limb amputation;, diabetes is a leading cause of "nontraumatic" lower limb amputation. ${ }^{8}$

There is wide agreement that DPN is a consequence of diabetes longstanding hyperglycemia which is critical for peripheral nerve damage and distal-predominant nerve fiber degeneration. ${ }^{9-11}$ The chronic diabetes hyperglycemia is compounded by numerous metabolic aberrations and diabetic microvascular complications resulting from disturbed nutritional support especially of the most distal parts of very long nerve axons originating in the spinal cord and travel long distances to supply the lower limbs and the feet, in particular. Additionally, the sparse vascular supply in this area, resulting from diabetes, is likely to cause further hypoxic nerve damage. Other confounding metabolic aberrations resulting from chronic hypoglycemia include the excessive release of cytokines, and exaggerated oxidative stress. This topic has been reviewed in detail elsewhere. 9 Although diabetes is considered the main underlying cause of DPN, the pre-diabetic stage with other comorbidities such as: obesity, hypertension and hyperlipidemia may increase the risk of neuropathy.${ }^{12-15}$ Moreover, treatment modalities received by diabetic patients (insulin and/or oral hypoglycemic agents) play an additional important role in delaying the development of this complication among diabetic patients. A meta-analysis conducted on 1,228 patients with type 1 diabetes and 6,669 patients with type 2 diabetes revealed significant reduction of neuropathy when blood sugar is controlled within the limits of normal. ${ }^{13}$

Type 2 diabetes mellitus is becoming a rapidly growing health problem in the affluent oil rich Gulf Council states (Saudi Arabia, Oman, Kuwait, Bahrain, and the United Arab Emirates). Its prevalence is highest in Saudi Arabia (31.6\%, 29.0\%.25.4\%. 25.0\%, 25.0\%, and $16.7 \%$, respectively). ${ }^{16}$ This high prevalence of type 2 diabetes was found to be significantly associated with the high Gross Domestic Product (GDP) and energy consumption. In contrast the lowest prevalence was found in the poorest Arab countries (Mauritania, 4.7\% and Somalia, 3.9\%).

Careful search in the literature uncovered only two small studies on painful DPN undertaken in different primary health facilities. ${ }^{17,18}$ The first study ${ }^{17}$ which was undertaken in a Primary Health Centre (PHC) in Riyadh found $35 \%$ of patients with type- 2 diabetes $(n=242)$ suffering from DPN. On the other hand, the second study ${ }^{18}$ which was also a PHC study found the prevalence of DPN to be $30.1 \%(n=235)$. With this alarming prevalence of DPN in Saudi diabetics we felt the need to have better documentation of DPN. Thus, the aim of the current study was to determine the prevalence and risk factors associated with the development of DPN in a large population of hospitalized adult Saudi diabetics.

\section{Methods}

This is a retrospective, nested case-control study conducted at King Abdulaziz Medical City (KAMC); a tertiary health care facility in Riyadh, Saudi Arabia. All adult patients, who were admitted with diabetes mellitus at KAMC, during the study period (from January 1 to December 31, 2018) were considered for inclusion in the study. Additionally, they met the ADA diagnosis criteria (fasting blood glucose $\geq 7.0 \mathrm{mmol} / \mathrm{L}$, glycosylated hemoglobin $[\mathrm{HbA1C}] \geq 6.5 \%$, classic symptoms of hyperglycemia, hyperglycemic crisis with a random plasma glucose $\geq 11.1 \mathrm{mmol} / \mathrm{L}$ or 2 -hour plasma glucose $\geq 11.11 \mathrm{mmol} / \mathrm{L}$ ). Of these, patients with confirmed DPN diagnosis were identified and served as case patients. Whereas, three controls per case were randomly selected from the remaining diabetic patients without peripheral neuropathy (PN). Patients who were under the age of 18 or did not fulfill the eligibility criteria were not considered for participation in the study. ${ }^{19}$ DPN confirmation was based on the following criteria: 1) abnormal clinical examination for pain, touch, vibration, pressure, power, and ankle reflex; and 2) abnormal nerve conduction studies (NCS) and/or electromyography.

Medical records of all enrolled patients (cases and controls) were accessed and the collected data were recorded in computerized format. These data included: demographic characteristics: age, gender, body mass index (BMI), type of diabetes, presence and duration of diabetic neuropathy, hypertension, kidney disease, dyslipidemia, and other comorbidities, life style (smoking and alcohol use), blood biochemistry indices (fasting blood glucose, HbA1C, lipid profile), medications, particularly insulin, oral hypoglycemic agents and diet, blood pressure and other clinical complications.

All methods and procedures were performed in accordance with the relevant institutional guidelines and regulations. The study was approved by the Institutional Review 
Board at King Abdullah International Medical Research Center (KAIMRC). The ethical committee waived the need for written informed consent due to the retrospective nature of the study.

\section{Statistical Analysis}

Data were analyzed using the Statistical Package for the Social Sciences Program (IBM SPSS Statistics version 24). Continuous data were presented as medians (interquartile range), and categorical data as frequencies (\%). Data were compared using Mann-Whitney $U$-test or $\chi^{2}$ test; as appropriate. Conditional logistic regression analysis was conducted to identify the factors associated with the DPN. Factors found statistically significant in logistic univariate analyses were included in the final multivariate model. Strength of the association was expressed as odds ratios (OR) with 95\% confidence interval. All tests were two-sided and a $p$-value less than 0.05 was considered statistically significant.

\section{Results}

A total of 2,906 patients with diabetes were identified during the study period. Of these, 73 patients $(3.5 \%)$ were found and labeled in the electronic chart by the treating clinician with DPN and 219 patients (three to one DPN case) were randomly selected as a control group. Table 1 shows the basic characteristics of the two groups. Females represented $51.8 \%$ and $63.0 \%$ of the DPN and the controls, respectively. The median (IQR) age of patients in the DPN group was significantly higher than in the controls [65 (58-75) vs 61 (45-71), $p=0.002)]$ and the duration of diabetes of patients with DPN was significantly higher than in the controls [8.5 (5.5-16) vs $6.5(4-15), p=0.031]$. Type 2 DM was diagnosed in $66(90.4 \%)$ patients with DPN, compared with $173(78.6 \%)$ patients in the control group, and 7 (9.6\%) patients with DPN were diagnosed with type 1 DM compared with $47(21.4 \%)$ patients in the control group. The median BMI of patients with DPN was significantly higher than controls [31 (2635 ) vs 27 (22-33), $p=0.003]$. However, the two groups were similar in gender distribution $(p=0.096)$, treatment modalities $(p=0.072)$, smoking status $(p=0.131)$ and alcohol consumption $(p=0.413)$. Biochemical indices and assessment parameters were also similar in both groups (Table 1).

The median number of comorbidities in DPN patients was significantly higher than in controls [5(4-7.5) vs 4 (2-
5.75), $p<0.001]$. Hypertension was more prevalent in the DPN patients $(p=0.005)$; so were peripheral vascular disease $(p<0.001)$, cerebral vascular accident $(p=0.027)$, chronic kidney disease $(p<0.001)$ and dyslipidemia $(p=0.002)$, than in control patients. Other comorbid diseases were similar in both groups. The DPN patients developed diabetic complications more than the control patients (Table 2).

Table 3 shows the results of the final multivariate logistic regression for factors associated with DPN. The independent factors associated with the likelihood of DPN were age (OR 1.02, 95\% CI 1.01-1.05, $p=0.031$ ), peripheral vascular disease (OR 3.14, 95\% CI 1.39-7.127, $p=0.006$ ) and chronic kidney disease (OR 2.39, 95\% CI $1.23-4.64, p=0.010)$. However, the presence of hypertension, cerebral vascular disease and dyslipidemia were not significantly associated with DPN.

\section{Discussion}

DPN is a very distressing chronic complication of diabetes with an array of poor outcomes. For example, DPN leads to neuropathic pain and diminished sensation which in turn can lead to frequent falls and injuries, restriction of movement, poor quality of life and difficulties with earning a living. Other serious complications include: leg ulcers that can eventually end in leg amputation. ${ }^{1-5,20,21}$

DPN is classified into numerous types and subtypes based on a mixture of phenomenological, aetiological, pathological and neurophysiological parameters. ${ }^{1,22-24}$ In a retrospective study, like the present one, it is difficult to assure the reliability of information on the details of these types or subtypes and be confident that such information has been documented in every patient. Accordingly, we restricted the definition of DPN, as mentioned earlier, to the presence of symptoms and/or signs of peripheral nerve dysfunction in patients with diabetes after the exclusion of other causes. ${ }^{1}$

Interest in DPN stems from the fact that it has recently been recognized as the most common of all the neuropathy complications worldwide. ${ }^{25}$ However, the reported prevalence of DPN varies widely from study to study and is heavily dependent on the type of diabetes, the population selected, and criteria used for defining cases, including the degree of control of the hyperglycemia. ${ }^{26}$ The most classical citation of DPN is a study conducted in France ${ }^{27}$ in which 4,400 adults with diabetes were followed for 36 years (1937-1973). Approximately 50\% of these patients developed DPN at the end of the follow-up period. Pirart ${ }^{27}$ found that two thirds of patients with diabetes had 
Table I Clinical and Demographic Characteristics of the DPN and Control Patients

\begin{tabular}{|c|c|c|c|}
\hline Variables & $\begin{array}{l}\text { Control } \\
(n=220)\end{array}$ & $\begin{array}{l}\text { DPN } \\
(n=73)\end{array}$ & $p$-value \\
\hline Age, years (median, IQR) & $6 I(45-7 I)$ & $65(58-75)$ & 0.002 \\
\hline Gender, n (\%) & & & 0.096 \\
\hline Male & $106(48.2)$ & $27(37.0)$ & \\
\hline Female & II4 (5I.8) & $46(63.0)$ & \\
\hline Duration of diabetes years, median (IQR) & $6.5(4-15)$ & $8.5(5.5-16)$ & 0.031 \\
\hline Type of DM, n (\%) & & & 0.025 \\
\hline Type I & $47(21.4)$ & $7(9.6)$ & \\
\hline Type II & $173(78.6)$ & $66(90.4)$ & \\
\hline Type of treatment, n (\%) & & & 0.072 \\
\hline Insulin & $158(72.8)$ & $62(86.1)$ & \\
\hline Oral hypoglycemic & $53(24.4)$ & $9(12.5)$ & \\
\hline Diet & $6(2.8)$ & I (I.4) & \\
\hline $\mathrm{BMI} \mathrm{kg} / \mathrm{cm}^{2}$, median (IQR) & $27(22-33)$ & $31(26-35)$ & 0.003 \\
\hline Smoking status & $29(13.2)$ & $15(20.5)$ & 0.131 \\
\hline Alcohol status & $2(0.9)$ & $0(0.0)$ & 0.413 \\
\hline \multicolumn{4}{|l|}{ Laboratory indices } \\
\hline Fasting blood glucose mmol/L (median, IQR) & $9(7-12)$ & $10(6-14)$ & 0.507 \\
\hline HbAIC (median, IQR) & $9(7-10)$ & $9(7-10)$ & 0.851 \\
\hline Systolic blood pressure mmHg (median, IQR) & $124(107-139)$ & $125(110-143)$ & 0.254 \\
\hline Diastolic blood pressure $\mathrm{mmHg}$ (median, IQR) & $69(58-76)$ & $66(55-73)$ & 0.158 \\
\hline Micro-albuminuria (median, IQR) & $26(2-100)$ & $50(50-200)$ & 0.092 \\
\hline Proteinuria (median, IQR) & $2(1.25-2)$ & $2(I-2)$ & 0.218 \\
\hline Serum Creatinine umol/L (median, IQR) & $7 \mid(6|-| 17)$ & $103(80-192.5)$ & $<0.001$ \\
\hline Cholesterol mmol/L (median, IQR) & $4(3-5)$ & $4(3-4.75)$ & 0.649 \\
\hline Triglycerides mmol/L (median, IQR) & $I(I-2)$ & $I(I-2)$ & 0.356 \\
\hline Albumin g/L (median, IQR) & $37(32-40)$ & $35(30-39)$ & 0.035 \\
\hline
\end{tabular}

Abbreviations: BMI, body mass index; DPN, diabetes peripheral neuropathy; IQR, interquartile range.

objective evidence of some form of neuropathy. The most common was DPN; affecting $50 \%$ of type 2 diabetics. Outside Europe, particularly in Asia and Africa, a very wide range of prevalences were reported. For examples Saudi Arabia $(19.9 ; \mathrm{n}=552) ;{ }^{28}$ Turkey $(60 \% ; \mathrm{n}=550),{ }^{29}$ China: $(61.8 \%, \mathrm{n}=435){ }^{30}$ and Tanzania $(72.2 \%, \mathrm{n}=$ 327). ${ }^{31}$ On the other hand, recent studies from Europe and the US have reported prevalences ranging from $6 \%$ to $51 \%$ depending on the population studied. ${ }^{1}$ We noted that the frequency of DPN in our patients was considerably lower than expected. This maybe owing to the fact that, typically, DPN is often diagnosed clinically with little further laboratory investigations to confirm the diagnosis or poorly captured in patients' records. In a study by Day et $\mathrm{al}^{32}$ more than $40 \%$ of diabetic patients in general practice had no biochemical evaluation, eye or foot examination. $^{32}$

The wide geographical variations in prevalence of DPN highlight the need for a global multicenter study in which the selection criteria for diabetic patients are unified to the finest detail. Hopefully, then, the true reflection of the geographical and ethnic differences in the prevalence of DPN can be meaningful and may shed some light in the mechanism underlying the differences in the prevalences in the DPN in different ethnic groups.

This possibility proved to be true in a comparative study between South Asians and Caucasians living in Britain where the prevalence of DPN was found to be significantly lower in South Asians (38.1\%) than White Caucasians (54.3\%). Further observations uncovered 
Table 2 Associated Comorbidities and Diabetes Complications

\begin{tabular}{|c|c|c|c|}
\hline Variables & $\begin{array}{l}\text { Control } \\
(n=220)\end{array}$ & $\begin{array}{l}\text { DPN } \\
(n=73)\end{array}$ & $p$-value \\
\hline Number of comorbid disease (median, IQR) & $4(2-5.75)$ & $5(4-7.5)$ & $<0.001$ \\
\hline Ischemic heart disease, n (\%) & $92(42)$ & $37(50.7)$ & 0.196 \\
\hline Congestive heart failure, $\mathrm{n}(\%)$ & $53(24.4)$ & $23(31.5)$ & 0.226 \\
\hline Chronic kidney disease, n (\%) & $32(14.6)$ & $27(37)$ & $<0.001$ \\
\hline Cerebral vascular stroke, $\mathrm{n}(\%)$ & $32(14.7)$ & $19(26)$ & 0.027 \\
\hline Hypertension, n (\%) & $160(73)$ & $65(89)$ & 0.005 \\
\hline Dyslipidemia, n (\%) & $119(54.6)$ & $553(75)$. & 0.002 \\
\hline Peripheral vascular disease, $\mathrm{n}(\%)$ & $15(7)$ & $17(24)$ & $<0.001$ \\
\hline Osteoarthritis, n (\%) & $15(6.9)$ & II (15) & 0.034 \\
\hline Hypoglycemia, n (\%) & $10(4.6)$ & $2(2.7)$ & 0.492 \\
\hline Diabetes ketoacidosis, n (\%) & $30(13.8)$ & $\mathrm{I}(\mathrm{l} .4)$ & 0.003 \\
\hline \multicolumn{4}{|l|}{ Diabetes complications, n (\%) } \\
\hline Retinopathy & $28(12.8)$ & $16(21.9)$ & 0.061 \\
\hline Foot ulcer & $15(6.9)$ & $13(17.8)$ & 0.006 \\
\hline Gangrene & $3(\mathrm{I} .4)$ & $7(9.6)$ & 0.001 \\
\hline Lower limb ischemia & $7(3.2)$ & $10(13.7)$ & 0.001 \\
\hline
\end{tabular}

Abbreviations: DPN, diabetes peripheral neuropathy; IQR, interquartile range.

foot insensitivity as assessed by $10 \mathrm{~g}$ monofilament perception, was more common in Caucasians $(43.9 \%$ vs $23.8 \%$ ). South Asians have better preserved small nerve fiber integrity than equivalent Europeans.
Corneal nerve fiber length $(22.0 \pm 7.9$ vs $19.3 \pm 6.3$ $\left.\mathrm{mm} / \mathrm{mm}^{2}\right)$, corneal nerve branch density [(geometric mean (range): 60.0 (4.7-246.2) vs 46.0 (3.1-129.2) no. $\left./ \mathrm{mm}^{2}\right]$, and heart rate variability [(geometric mean

Table 3 Univariate and Multivariate Conditional Logistic Regression for Risk Factors Associated with DPN

\begin{tabular}{|c|c|c|c|c|}
\hline \multirow[t]{2}{*}{ Factor } & \multicolumn{2}{|c|}{ Univariate Analysis } & \multicolumn{2}{|c|}{ Multivariate Analysis } \\
\hline & OR (95\% CI) & $p$-value & OR (95\% CI) & $p$-value \\
\hline Age & $1.03(1.01-1.05)$ & $<0.001$ & $1.02(1.01-1.04)$ & 0.031 \\
\hline $\begin{array}{l}\text { Chronic kidney disease } \\
\text { Yes } \\
\text { No }\end{array}$ & $\begin{array}{l}3.43(1.87-6.28) \\
1\end{array}$ & $<0.001$ & $\begin{array}{l}2.39(1.23-4.64) \\
1\end{array}$ & 0.010 \\
\hline $\begin{array}{l}\text { Cerebral vascular stroke } \\
\text { Yes } \\
\text { No }\end{array}$ & $\begin{array}{l}2.05(1.08-3.89) \\
1\end{array}$ & 0.029 & $\begin{array}{l}1.64(0.80-3.33) \\
I\end{array}$ & 0.176 \\
\hline $\begin{array}{l}\text { Peripheral vascular disease } \\
\text { Yes } \\
\text { No }\end{array}$ & $\begin{array}{l}4.11(1.93-8.74) \\
1\end{array}$ & $<0.001$ & $\begin{array}{l}3.14(1.39-7.13) \\
1\end{array}$ & 0.006 \\
\hline $\begin{array}{l}\text { Hypertension } \\
\text { Yes } \\
\text { No }\end{array}$ & $\begin{array}{l}3.00(1.36-6.62) \\
1\end{array}$ & 0.007 & $\begin{array}{l}083(0.27-2.54) \\
\mathrm{I}\end{array}$ & 0.740 \\
\hline $\begin{array}{l}\text { Dyslipidemia } \\
\text { Yes } \\
\text { No }\end{array}$ & $\begin{array}{l}2.54(\mathrm{I} .40-4.6 \mathrm{I}) \\
\mathrm{I}\end{array}$ & 0.002 & $\begin{array}{l}\text { I. } 56(0.78-3 \mid 3) \\
\mid\end{array}$ & 0.181 \\
\hline
\end{tabular}

Abbreviations: $\mathrm{Cl}$, confidence interval; OR, odds ratio. 
(range): 7.9 (1.4-27.7) vs 6.5 (1.5-22.0)], were significantly higher in South Asians than Europeans. ${ }^{33,34}$

As mentioned earlier DPN is believed to be a consequence of the diabetes longstanding hyperglycemia which is critical for peripheral nerve damage and distal-predominant nerve fiber degeneration. ${ }^{9-11}$ The hyperglycemia of the chronic diabetes process is compounded by numerous metabolic aberrations and diabetic microvascular complications resulting from the disturbed nutritional support especially of the most distal parts of very long nerve axons originating in the spinal cord and travel long distances to supply the lower limbs and the feet in particular. Additionally, the sparse vascular supply in this area resulting from diabetes is likely to cause further hypoxic nerve damage. Other confounding metabolic aberrations resulting from the chronic hypoglycemia include excessive release of cytokines, and exaggerated oxidative stress. This topic has been reviewed in detail elsewhere. ${ }^{9}$

It is also becoming clear that the duration of diabetes and glycemic control are the most significant risk factors for DPN. ${ }^{35}$ In regard to the benefit of blood glucose control, there are reports indicating that aggressive blood glucose control was associated with lower prevalence of DPN. ${ }^{36-39}$ In support of this notion, in the current study, we found an association between DPN and blood glucose control as reflected by the fasting blood glucose level, $\mathrm{HbAlc}$ and the type of treatment. Therefore, like others ${ }^{40}$ the most effective approach to reduce the progression to neuropathy is aggressive treatment of diabetes hyperglycemia towards normoglycemia.

Our finding that multiple risk factors are associated with the development of DPN in diabetic patients should direct the attention towards the link between these risk factors and the increase in diabetes-related complications, morbidity as well as mortality. ${ }^{40,41}$ It was also clear that age is a significant risk factor that predisposes to DPN and this finding was observed by others. ${ }^{1,20-22}$ In the present study the vast majority of patients with DPN were type 2 diabetes (90.4\%). Similarly, Kästenbauer et al, ${ }^{23}$ showed a higher percentage (16\% vs $37.5 \%)$ of type 2 diabetic patients with symptoms of neuropathy compared with type 1 diabetic patients. ${ }^{23}$ Other risk factors including BMI, number of comorbid diseases such as chronic kidney disease, hypertension, dyslipidemia, cerebral vascular stroke, peripheral vascular disease and diabetic ketoacidosis were also found to be significantly associated with DPN. The close association between DPN and modifiable cardiovascular risk factors (cardiometabolic disease) including elevated triglyceride levels (hyperlipidemia), body mass index (BMI), smoking, hypertension and cardiovascular disease was documented repeatedly in previous studies. ${ }^{1,20,21,24,42}$ However, no specific mechanisms linking DPN with cardiovascular disease have been suggested in any of these studies.

It also worth adding that the findings of the present study are consistent with those reported from the neighboring Gulf state of Bahrain. The Bahraini study ${ }^{22}$ found that older age, poor glycemic control, longer duration of diabetes, elevated cholesterol levels, current smoking, obesity, large waist circumference, elevated triglycerides levels and hypertension but not gender were significant risk factors for DPN. However, the vast majority of the DPN patients in the study were females and were on insulin therapy. In contrast, there were no significant differences in gender distribution or treatment modalities when compared to controls. On the other hand, many other studies have reported a significant association between female gender and the prevalence of DPN. ${ }^{1,20,21,24}$

In the multivariate model, we identified three risk factors that independently correlate with the presence of DPN. These were age, chronic kidney disease and peripheral vascular disease. These risk factors were closely associated with DPN and this association has been reported before. $^{1,24,26,37-43}$

However, we did not find any correlation between smoking and DPN. ${ }^{24,26,44}$ Nonetheless, the following diseases were found to be associated with DPN: PVD, kidney disease, hypertension, dyslipidemia, foot ulcer and amputation, which is in line with many earlier studies. ${ }^{15,37,39,44,45}$

Our findings concur with those reported in recent published studies on the factors associated with DPN. These studies were noticeable multi-center studies of long-term follow-up of 20-25 years. ${ }^{46,47}$ Most of these studies confirmed that old age, longer diabetes duration, and elevated $\mathrm{HbAlc}$ were the commonest risk factors for the development of DPN. ${ }^{45-48}$

We acknowledge several limitations in our study. The nature of study design as a retrospective study limits the accurate assessment of the association between DPN and risk factors. Therefore, prospective studies are needed to fully characterize the sequel of DPN in diabetes patients. Poor documentation of DPN diagnosis and supportive laboratories investigations (nerve conduction studies and electromyography) reduced our ability to access important data needed for the study and to confirm how DPN 
diagnosis was made. Therefore, it is likely that some DPN patients were missed.

\section{Conclusion}

This study identified several risk factors that contributed to the development of DPN in Saudis. These must be considered in strategies and campaigns aimed at risk reduction of cardiovascular and chronic diseases, and consequently progression of DPN.

\section{Ethics}

The study was approved by the Institutional Review Board, King Abdullah International Medical Research Centre, King Saud bin Abdulaziz University for Health Sciences, National Guard Health Affairs. All patient data accessed complied with relevant data protection and privacy regulations. All procedures performed in the study involving human participants were in accordance with the ethical standards of the institutional and/or national research committee and with the 1964 Helsinki declaration and its later amendments or comparable ethical standards.

\section{Acknowledgments}

We would like to acknowledge the help of the Health Informatics Department, KAMC.

\section{Author Contributions}

All authors made substantial contributions to conception and design, acquisition of data, or analysis and interpretation of data; took part in drafting the article or revising it critically for important intellectual content; agreed to submit to the current journal; gave final approval of the version to be published; and agree to be accountable for all aspects of the work.

\section{Funding}

There is no funding to report.

\section{Disclosure}

The authors report no conflicts of interest for this work.

\section{References}

1. Hicks CW, Selvin E. Epidemiology of peripheral neuropathy and lower extremity disease in diabetes. Curr Diab Rep. 2019;19:86. doi: doi:10.1007/s11892-019-1212-8.

2. Shiferaw WS, Akalu YT, Work Y, Yared Asmare Aynalem YA. Prevalence of diabetic peripheral neuropathy in Africa: a systematic review and meta-analysis. MBC Endoc Disord. 2020;20:49-58. doi:10.1186/s12902-020-0534-5
3. Alleman CJ, Westerhout KY, Hensen M, et al. Humanistic and economic burden of painful diabetic peripheral neuropathy in Europe: a review of the literature. Diabet Res Clin Pract. 2015;109:215-225. doi:10.1016/j.diabres.2015.04.031

4. Ebata-Kogure N, Nozawa K, Murakami A, Toyoda T. Clinical and economic burdens experienced by patients with painful diabetic peripheral neuropathy: an observational study using a Japanese claims database. PLoS One. 2017;12(10):e0187250. doi:10.1371/journal. pone. 0187250

5. John J, Stamenitis S, Clouth J, et al. The quality of life and economic burden of neuropathy in diabetic patients in Germany in 2002-results from the Diabetic Microvascular Complications (DIMICO) study. Diabetes Res Clin Pract. 2008;81:223-230. doi:10.1016/j. diabres.2008.03.019

6. Ugwu E, Adeleye O, Ezeani I. Predictors of lower extremity amputation in patients with diabetic foot ulcer: findings from MEDFUN, a multi-center observational study. J Foot Ankle Res. 2019;12:34. doi:10.1186/s13047-019-0345-y

7. Kuntzer T, Medlin F, Burnand B, Camain JY. Diabetic neuropathies: clinical sub-types, early detection, and asking help from neurologist. Praxis (Bern 1994). 2012;101(20):1315-1319. doi:10.1024/16618157/a001037

8. Lazzarini PA, Damien Clark D, Derhy PH. What are the major causes of lower limb amputations in a major Australian teaching hospital? The Queensland Diabetic Foot Innovation Project, 2006 - 2007. J Foot Ankle Res. 2011;4(Suppl1):O24. doi:10.1186/1757-1146-4-S1-O24

9. Yagihashi S, Mizukami H, Sugimoto K. Mechanism of diabetic neuropathy: where are we now and where to go? A review. $J$ Diabetes Invest. 2011;2:18-32. doi:10.1111/j.2040-1124.2010.00070

10. Boulton A, Malik R, Arezzo J, Sosenko J. Diabetic somatic neuropathies. Diabetes Care. 2004;27(6):1458-1486. doi:10.2337/ diacare.27.6.1458

11. Won J, Kwon H, Kim C, et al. Prevalence and clinical characteristics of diabetic peripheral neuropathy in hospital patients with Type 2 diabetes in Korea. Diabetic Med. 2012;29(9):e290. doi:10.1111/ j.1464-5491.2012.03697.x

12. Boronikolos GC, Menge BA, Schenker N, et al. Upper gastrointestinal motility and symptoms in individuals with diabetes, prediabetes and normal glucose tolerance. Diabetologia. 2015;58(6):1175-1182. doi:10.1007/s00125-015-3538-3

13. Callaghan BC, Little AA, Feldman EL, Hughes RA. Enhanced glucose control for preventing and treating diabetic neuropathy. Cochrane Database Syst Rev. 2012;6:CD007543.

14. Tesfaye S, Stevens L, Stephenson J, et al. Prevalence of diabetic peripheral neuropathy and its relation to glycaemic control and potential risk factors: the EURODIAB IDDM Complications Study. Diabetologia. 1996;39(11):1377-1384. doi:10.1007/ s001250050586

15. Tesfaye S, Chaturvedi N, Eaton SE, et al. Vascular risk factors and diabetic neuropathy. $N$ Engl $J$ Med. 2005;352(4):341-350. doi:10.1056/NEJMoa032782

16. Meo SA, Usmani AM, Qalbani E. Prevalence of type 2 diabetes in the Arab world: impact of GDP and energy consumption. Europ Med Pharmacol Sci. 2017;21:1303-1312.

17. Algeffari MA. Painful diabetic peripheral neuropathy among Saudi diabetic patients is common but under-recognized: multicenter crosssectional study at primary health care setting. Family Community Med. 2018;25(1):43-47.

18. Sendi RA, Mahrus A, Saeed RM, et al. Diabetic peripheral neuropathy among Saudi diabetic patients: a multicenter cross-sectional study at primary health care setting. J Family Med Prim Care. 2020;9 (1):197-201. doi:10.4103/jfmpc.jfmpc_927_19

19. American Diabetes Association. "Diagnosis and classification of diabetes mellitus.". Diabetes Care. 2010;33(supplement 1):S62S69. doi:10.2337/dc10-S062 
20. Yang H, Sloan G, Ye Y, Wang S. New perspective in diabetic neuropathy: from the periphery to the brain, a call for early detection, and precision medicine. Front Endocrinol. 2020;10. doi:10.3389/ fendo.2019.00929

21. Dyck PJ, Kratz KM, Karnes JL, et al. The prevalence by staged severity of various types of diabetic neuropathy, retinopathy, and nephropathy in a population-based cohort: the Rochester diabetic neuropathy study. Neurology. 1993;43:817-824. doi:10.1212/ WNL.43.4.817

22. Al-Mahroos F, Al-Roomi K. Diabetic neuropathy, foot ulceration, peripheral vascular disease and potential risk factors among patients with diabetes in Bahrain: a nationwide primary care diabetes clinicbased study. Ann Saudi Med. 2007;27(1):25.

23. Albers JW, Pop-Busui R. Diabetic neuropathy: mechanisms, emerging treatments, and subtypes. Review. Curr Neurol Neurosci Rep. 2014;14:473. doi:10.1007/s11910-014-0473-3

24. Pop-Busui R, Boulton AJM, Feldman EL, Bril V. Diabetic Neuropathy: A Position Statement by the American Diabetes Association. Diabetes Care. 2017;40:136-154. doi:10.2337/dc16-2042

25. Iqbal Z, Azmi S, Yadav R, et al. Diabetic peripheral neuropathy: epidemiology, diagnosis, and pharmacotherapy. Clin Ther. 2018;40:828-849. doi:10.1016/j.clinthera.2018.04.001

26. Ziegler D, Papanas N, Vinik AI, Shaw JE. Epidemiology of polyneuropathy in diabetes and prediabetes. Handb Clin Neurol. 2014;126::3-22. doi:10.1016/B978-0-444-53480-4.00001

27. Pirart J. [Diabetes mellitus and its degenerative complications: a prospective study of 4400 patients observed between 1947 and 1973 (3rd and last part) (author's transl)]. Diabete Metab. 1977;3:2-4. French.

28. Wang DD, Bakhotmah BA, Hu FB, Alzahrani HA. Prevalence and correlates of diabetic peripheral neuropathy in a Saudi Arabic population: a cross-sectional study. PLoS One. 2014;9:e106935. doi:10.1371/journal.pone.0106935

29. Börü UT, Alp R, Sargin H, et al. Prevalence of peripheral neuropathy in type 2 diabetic patients attending a diabetes center in Turkey. Endocr J. 2004;51:563-567.

30. Lu B, Yang Z, Wang M, et al. High prevalence of diabetic neuropathy in population-based patients diagnosed with type 2 diabetes in the Shanghai downtown. Diabetes Res Clin Pract. 2010;88(2010):289294. doi:10.1016/j.diabres.2010.02.002

31. Amour AA, Chamba N, Kayandabila J, Lyaruu IA. Prevalence, patterns, and factors associated with peripheral neuropathies among diabetic patients at tertiary hospital in the Kilimanjaro region: descriptive cross-sectional study From North-Eastern Tanzania. Int J Endocrinol. 2019;2019:5404781. doi:10.1155/2019/5404781

32. Day JL, Humphreys H, Alban-Davies H. Problems of comprehensive shared diabetes care. Br MedJ. 1987;294:1590-1592. doi:10.1136/ bmj.294.6587.1590

33. Tahrani AA, Altaf QA, Piya MK, Barnett AH. Peripheral and autonomic neuropathy in south asians and white caucasians with type 2 diabetes mellitus: possible explanations for epidemiological differences. J Diabetes Res. 2017;2017:1273789. doi:10.1155/2017/ 1273789.

34. Fadavi H, Tavakoli M, Foden P, et al. Explanations for less small fibre neuropathy in South Asian versus European subjects with type 2 diabetes in the UK. Diabetes Metab Res Rev. 2018;34:e3044. doi:10.1002/dmrr.3044.
35. Callaghan BC, Gao L, Li Y, et al. Diabetes and obesity are the main metabolic drivers of peripheral neuropathy. Ann Clin Transl Neurol. 2018;5:397-405. doi:10.1002/acn3.531.

36. Ang L, Jaiswal M, Martin C, et al. Glucose control and diabetic neuropathy: lessons from recent large clinical trials. Curr Diab Rep. 2014;14(528). doi:10.1007/s11892-014-0528-7

37. Harris M, Eastman R, Cowie C. Symptoms of sensory neuropathy in adults with NIDDM in the U.S. population. Diabetes Care. 1993;16:1446-1452. doi:10.2337/diacare.16.11.1446

38. Al-Kaabi JM, FAl M, Zoubeidi T, et al. Prevalence and determinants of peripheral neuropathy in patients with type 2 diabetes attending a tertiary care center in the United Arab Emirates. $J$ Diabetes Metab. 2014;5(346):2.

39. Cameron NE, Eaton SE, Cotter MA, Tesfaye S. Vascular factors and metabolic interactions in the pathogenesis of diabetic neuropathy. Diabetologia. 2001;44:1973-1988. doi:10.1007/s001250100001

40. Andersen ST, Witte DR, Dalsgaard EM, et al. Risk factors for incident diabetic polyneuropathy in a cohort with screen-detected type 2 diabetes followed for 13 years: ADDITION-denmark. Diabetes Care. 2018;41:1068-1075. doi:10.2337/dc17-2062.

41. Abbott CA, Malik RA, van Ross ER, et al. Prevalence and characteristics of painful diabetics. Explanations for less small fibre neuropathy in a large community-based diabetic population South Asian versus European subjects with type 2 diabetes in the UK. Diabetes Care. 2011;34(10):2220-2224. doi:10.2337/dc11-1108

42. Yang CP, Lin CC, Li CI, et al. Cardiovascular risk factors increase the risks of diabetic peripheral neuropathy in Patients with type 2 diabetes mellitus: the Taiwan diabetes study. Medicine (Baltimore). 2015;94:e1783. doi:10.1097/MD.0000000000001783

43. Kiani J, Moghimbeigi A, Azizkhani H, Kosarifard S. The prevalence and associated risk factors of peripheral diabetic neuropathy in Hamedan, Iran. Arch Iran Med. 2013;16:17-19.

44. Kästenbauer T, Irsigler P, Sauseng S, Grimm A, Prager R. The prevalence of symptoms of sensorimotor and autonomic neuropathy in Type 1 and Type 2 diabetic subjects. J Diabetes Complications. 2004;18:27-31. doi:10.1016/S1056-8727(03)00071-0

45. Coppini DV, Bowtell PA, Weng C, Young PJ, Sonksen PH. Showing neuropathy is related to increased mortality in diabetic patients a survival analysis using an accelerated failure time model. J Clin Epidemiol. 2000;53:519-523. doi:10.1016/S0895-4356(99)00170-5

46. Braffett BH, Gubitosi-Klug RA, Albers JW, et al. Risk Factors for diabetic peripheral neuropathy and cardiovascular autonomic neuropathy in the Diabetes Control and Complications Trial/Epidemiology of Diabetes Interventions and Complications (DCCT/EDIC) Study. The DCCT/EDIC and Research Group*. Diabetes. 2020;69:10001010. doi:10.2337/db19-1046

47. Pfannkuche A, Alhajjar A, Ming A, et al. Prevalence and risk factors of diabetic peripheral neuropathy in a diabetics cohort: register initiative "diabetes and nerves ". Endoc Metab Sci. 2020;1(12):100053. doi:10.1016/j.endmts.2020.100053

48. Pan Q, Li Q Q, Deng W, et al. Prevalence of and risk factors for peripheral neuropathy in Chinese patients with diabetes: a multicenter cross-sectional study. Front Endocrinol. 2018;9:Article 617. doi:10.3389/fendo.2018.00617 


\section{Publish your work in this journal}

The International Journal of General Medicine is an international, peer-reviewed open-access journal that focuses on general and internal medicine, pathogenesis, epidemiology, diagnosis, monitoring and treatment protocols. The journal is characterized by the rapid reporting of reviews, original research and clinical studies

across all disease areas. The manuscript management system is completely online and includes a very quick and fair peer-review system, which is all easy to use. Visit http://www.dovepress.com/ testimonials.php to read real quotes from published authors.

Submit your manuscript here: https://www.dovepress.com/international-journal-of-general-medicine-journal 